\title{
Long-Term Effect of Ferric Citrate Hydrate on Mineral Metabolism and Anemia in Maintenance Hemodialysis Patients
}

\author{
Yuta Bando1, Mizuki Komatsu1, Masayuki Okazaki', Hiroshi Kawaguchi', Kosaku Nitta2* \\ ${ }^{1}$ Department of Nephrology, Jyoban Hospital, Fukushima, Japan \\ ${ }^{2}$ Department of Nephrology, Tokyo Women's Medical University, Tokyo, Japan \\ Email: *knitta@twmu.ac.jp
}

How to cite this paper: Bando, Y., Komatsu, M., Okazaki, M., Kawaguchi, H. and Nitta, K. (2020) Long-Term Effect of Ferric Citrate Hydrate on Mineral Metabolism and Anemia in Maintenance Hemodialysis Patients. International Journal of Clinical Medicine, 11, 719-730. https://doi.org/10.4236/ijcm.2020.1111053

Received: September 23, 2020

Accepted: November 27, 2020

Published: November 30, 2020

Copyright $\odot 2020$ by author(s) and Scientific Research Publishing Inc. This work is licensed under the Creative Commons Attribution International License (CC BY 4.0).

http://creativecommons.org/licenses/by/4.0/ (c) (i) Open Access

\begin{abstract}
Background: Ferric citrate hydrate is a phosphate (P)-binder drug that is indicated for patients with chronic kidney disease (CKD) undergoing maintenance hemodialysis (MHD). Objectives: The objectives of this study were to evaluate the changes in the serum mineral metabolism biomarker levels and treatment status of anemia in MHD patients receiving ferric citrate hydrate. Methods: A total of 132 adult dialysis patients were enrolled in this study. Bone turnover marker levels, anemia status and iron biomarker levels over a period of 18 months after the start of the ferric citrate hydrate treatment were extracted from the medical records of the patients. Results: At enrollment, 14 (10.6\%) patients were P-binder-naïve, and 118 (89.4\%) patients were receiving other P-binders. The serum P level before the start of ferric citrate hydrate treatment was $\leq 5.5 \mathrm{mg} / \mathrm{dL}$ in $18.8 \%$ of the patients. After 18 months of ferric citrate hydrate treatment, the proportion of patients with serum P levels $\leq 5.5$ $\mathrm{mg} / \mathrm{dL}$ increased to $47.7 \%$. The mean \pm standard error of the mean (SEM) of the serum P level was $7.05 \pm 0.14 \mathrm{mg} / \mathrm{dL}$ at the baseline, and decreased to 5.83 $\pm 0.17 \mathrm{mg} / \mathrm{dL}$ after 18 months of treatment with ferric citrate hydrate. The mean \pm SEM of the serum hemoglobin $(\mathrm{Hb})$, ferritin, and transferrin saturation (TSAT) levels were $11.96 \pm 0.76 \mathrm{~g} / \mathrm{dL}, 71.13 \pm 6.09 \mathrm{ng} / \mathrm{mL}$, and $25.19 \% \pm 1.21 \%$, respectively, at the baseline, and $11.45 \pm 0.13 \mathrm{~g} / \mathrm{dL}, 124.94 \pm 8.62 \mathrm{ng} / \mathrm{mL}$, and $26.18 \% \pm 0.99 \%$, respectively, after 18 months of ferric citrate hydrate treatment. Conclusions: These results suggest that the serum $\mathrm{P}$ and anemia biomarker levels observed in this study were similar to those reported in clinical trials.
\end{abstract}

\section{Keywords}

Ferric Citrate Hydrate, Hemodialysis, Mineral Metabolism, Anemia 


\section{Introduction}

Hyperphosphatemia is a common complication in patients with chronic kidney disease (CKD), particularly in those requiring dialysis therapy [1]. If left untreated, hyperphosphatemia has been reported to be associated with an increase in both the morbidity and mortality rates [2] [3]. Therefore, safe and effective use of phosphate $(\mathrm{P})$-binders is essential in the treatment of CKD patients undergoing dialysis.

In Japan, ferric citrate hydrate $\left(\right.$ Riona $\left.^{\oplus}\right)$ was approved in 2014 for use as a drug to reduce the serum P levels in dialysis patients [4]. The safety and efficacy of ferric citrate hydrate in dialysis patients had been examined in a 12-week randomized phase III study, in which ferric citrate hydrate was compared with sevelamer hydrochloride [5]; the study revealed that ferric citrate hydrate treatment resulted in a sustained reduction of the serum P level, to a degree comparable to that observed with sevelamer hydrochloride treatment. In addition, ferric citrate hydrate also exhibited the same safety profile and indication as sevelamer hydrochloride.

The objectives of this study were to examine the impact of ferric citrate hydrate treatment on the serum levels of mineral metabolism biomarkers and anemia management status in maintenance hemodialysis (MHD) patients who were started on ferric citrate hydrate treatment (P-binder-naïve) or were switched to the treatment from another P-binder drug.

\section{Methods}

\subsection{The Study Group}

This study was a single-center, prospective cohort study conducted over 18 months in Japan. The participants were MHD patients who had been undergoing hemodialysis (HD) via an arteriovenous fistula (AVF) at dialysis unit of Jyoban Hospital. The following patients were excluded at the time of enrollment: patients who were receiving other iron supplements; patients with obvious hemorrhagic symptoms, chronic inflammatory diseases or malignant tumors; patients with serum ferritin levels of $\geq 500 \mathrm{ng} / \mathrm{mL}$, in whom iron overload could not be ruled out. This study protocol was in compliance with the Declaration of Helsinki and was approved by the Institutional Review Board of Jyoban Hospital (No. 26-1). All subjects gave informed consent to participate.

\subsection{Assessment of Laboratory Data}

Baseline data included the gender, age, weight, comorbidities (diabetes, hypertension), dialysis vintage, and types of P-binders received. Clinicians collected the data on ferric citrate hydrate treatment, including the treatment period, mean number of tablets taken per day in each month and concomitant use of other P-binders, from the patients' medical records. Laboratory data on the serum P, calcium (Ca) and intact parathyroid hormone (iPTH) levels measured every 3 
months from the start of the ferric citrate hydrate treatment were collected (baseline: 0 month). In addition, data on the serum ferritin, transferrin saturation (TSAT) and hemoglobin $(\mathrm{Hb})$ levels measured every 3 months from the start of ferric citrate hydrate treatment were also collected.

Whether the collected test data might indicate the possible development of adverse events (AEs) or serious AEs was determined based on the normal ranges of serum ferritin, TSAT and $\mathrm{Hb}$ proposed by the Kidney Disease Improving Global Outcomes (KDIGO) guideline [6], and the normal ranges of serum P, Ca and iPTH proposed by the National Kidney Foundation's Kidney Disease Outcomes Quality Initiative (KDQOI) [6].

\subsection{Statistical Analysis}

The descriptive data and test values are expressed as the means \pm SEM. Data were examined by one-way analysis of variance, followed by Bonferroni's multiple comparison test. The statistical significance level was set at $\mathrm{P}<0.05$.

\section{Results}

A total of 139 patients were enrolled in the study, and with 7 dropping out during the course of the study, the remaining 132 patients were included in the analyses. Demographic and baseline characteristics are shown in Table 1. Male patients accounted for $59.8 \%$, and the mean age was 63.3 years. The dialysis vintage varied from 0 to 381 months, with a mean of 63.8 months. Before receiving the ferric citrate hydrate treatment, most patients (89.4\%) were already on treatment with other P-binders (prior P-binder therapy(+) subgroup), and the proportion of previously untreated patients (prior P-binder therapy(-) subgroup) was as low as $10.6 \%$.

Table 2 shows the P-binder drugs that had been used in the patients before the start of ferric citrate hydrate treatment. Patients who were using other P-binder drugs during the ferric citrate hydrate treatment were also counted. Of the patients, $10.6 \%$ (14 patients) had no previous history of treatment with P-binders patients (prior P-binder therapy $(-)$ subgroup). In the prior P-binder therapy $(+)$ subgroup (118 patients), 48.3\% of patients were receiving calcium carbonate monotherapy, and $19.5 \%$ were receiving a combination of calcium carbonate and lanthanum carbonate as prior P-binder therapy.

The mean number of tablets of ferric citrate hydrate prescribed to the patients was 6/day at the baseline, but increased to 7/day at 3 months and 6 months after the start. Examined separately in the prior P-binder therapy $(+)$ and prior Pbinder therapy(-) subgroups, the mean numbers of ferric citrate hydrate tablets prescribed were 6/day and 5/day, respectively, at the baseline, and 7/day and 6/day, respectively, at 6 months after the start of treatment. In 17 patients (18\%), ferric citrate hydrate was started as add-on treatment to the ongoing P-binder therapy.

Data on the serum P, Ca and iPTH levels were collected as the bone turnover 
Table 1. Demographic and baseline characteristics.

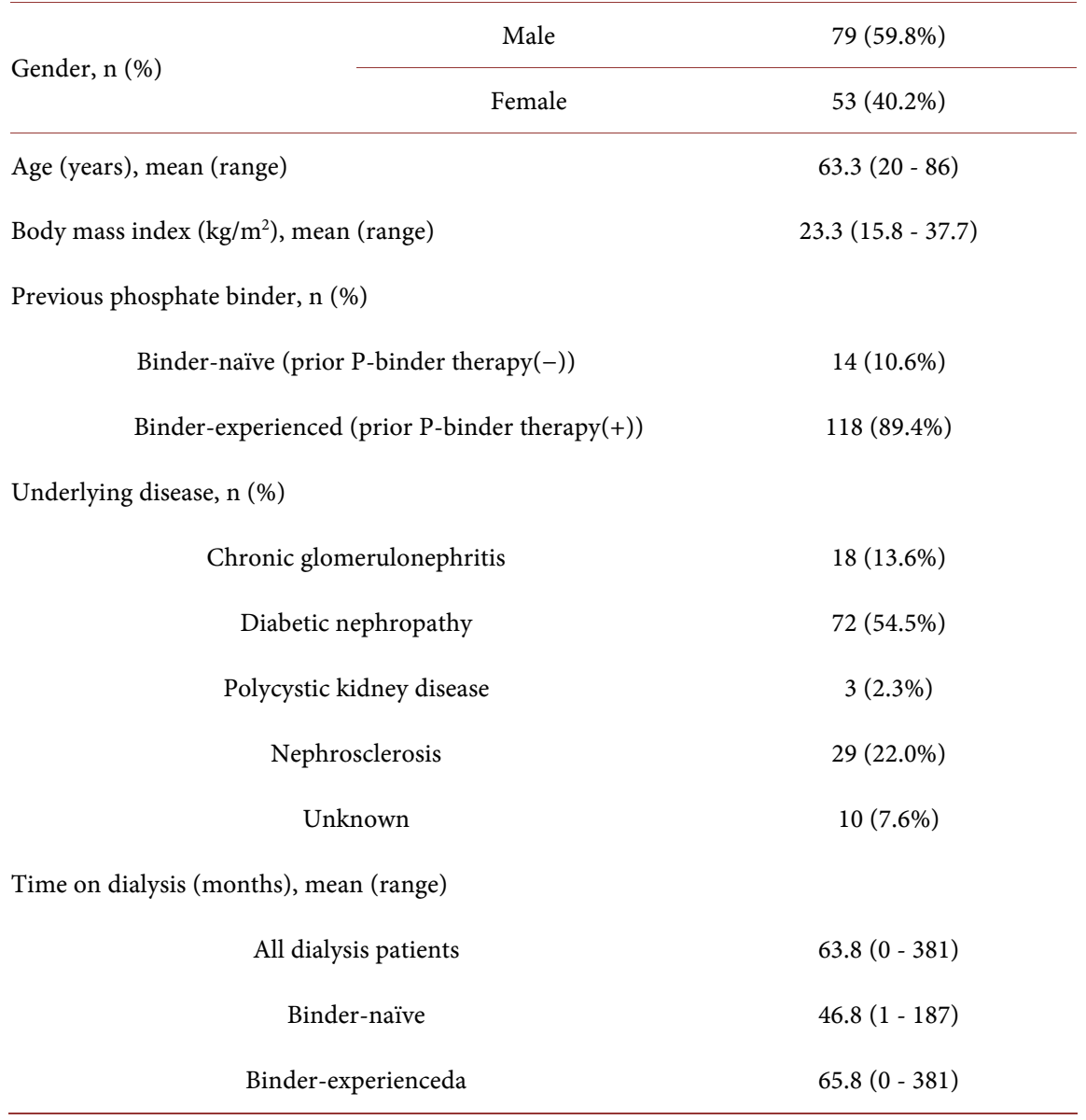

Table 2. History of phosphate binder use.

\begin{tabular}{cc}
\hline Type of previous phosphate binder & Binder-experienced $\mathrm{n}=118$ \\
Calcium only & $57(48.3 \%)$ \\
Lanthanum + Calcium & $23(19.5 \%)$ \\
Bixalomer + Calcium & $9(7.6 \%)$ \\
Lanthanum & $8(6.8 \%)$ \\
Lanthanum + Sevelamer + Calcium & $6(5.1 \%)$ \\
Bixalomer + Lanthanum + Calcium & $6(5.1 \%)$ \\
Sevelamer + Calcium & $3(2.5 \%)$ \\
Bixalomer + Lanthanum & $2(1.7 \%)$ \\
Sevelamer + Calcium & $2(1.7 \%)$ \\
Bixalomer + Lanthanum + Sevelamer + Calcium & $1(0.8 \%)$ \\
Bixalomer + Sevelamer + Calcium & $1(0.8 \%)$ \\
\hline
\end{tabular}


markers. Figure 1(a) shows the proportions of patients in whom the KDOQI target value of $\mathrm{P}(\mathrm{P} \leq 5.5 \mathrm{mg} / \mathrm{dL})$ was reached before and during the ferric citrate hydrate treatment. The proportion of patients with serum $P$ levels within the above target range was $18.8 \%$ at baseline, $44 \%$ at 3 months and $60 \%$ at 9 months after the start of ferric citrate hydrate treatment. The mean serum $\mathrm{P}$ value in the patients at the baseline was $7.05 \pm 0.14 \mathrm{mg} / \mathrm{dL}$, decreasing to $5.61 \pm 0.15 \mathrm{mg} / \mathrm{dL}$ after 6 months of treatment, and $5.83 \pm 0.17 \mathrm{mg} / \mathrm{dL}$ after 18 months of treatment (Figure 1(b), Table 3). The baseline mean serum P level was $6.80 \pm 0.56 \mathrm{mg} / \mathrm{dL}$ in the prior P-binder therapy(-) subgroup and $7.08 \pm 0.14 \mathrm{mg} / \mathrm{dL}$ in the prior P-binder therapy $(+)$ subgroup, being slightly lower in the prior P-binder therapy(-) subgroup (Table 3 ). However, serum $P$ levels in both groups decreased by the same extent from 1 month to 6 months after the start of ferric citrate hydrate treatment, with the levels remaining sustained at the reduced levels thereafter (Figure $1(\mathrm{c})$ ). The mean serum $\mathrm{Ca}$ level showed no change from the baseline in the patients, whereas the serum iPTH level tended to decrease gradually (Table 4).

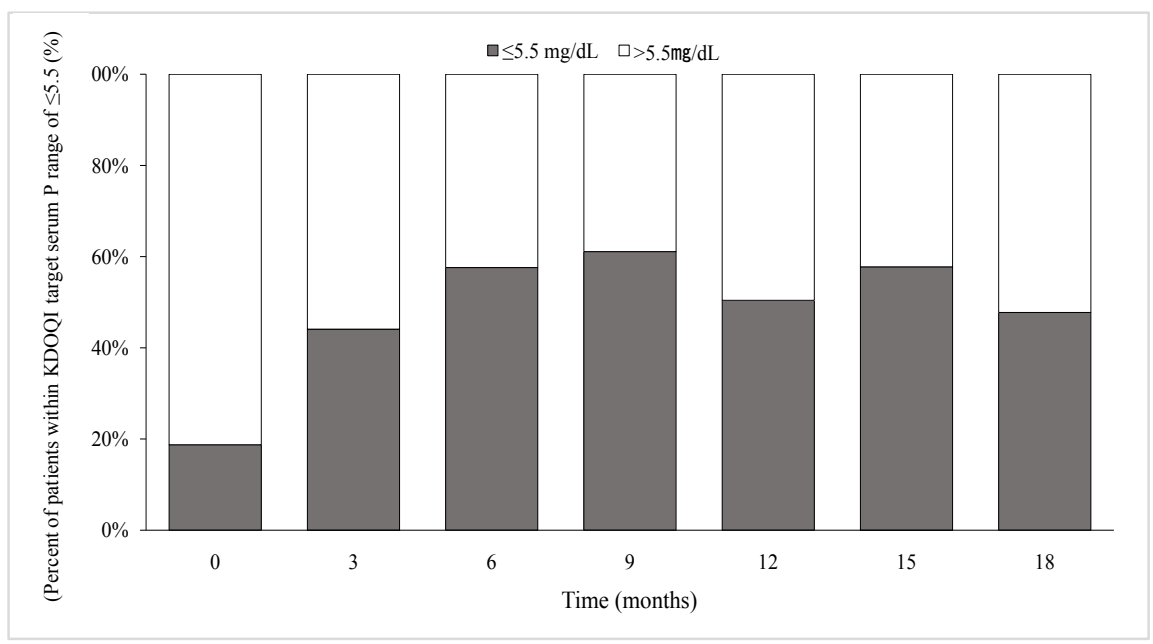

(a)

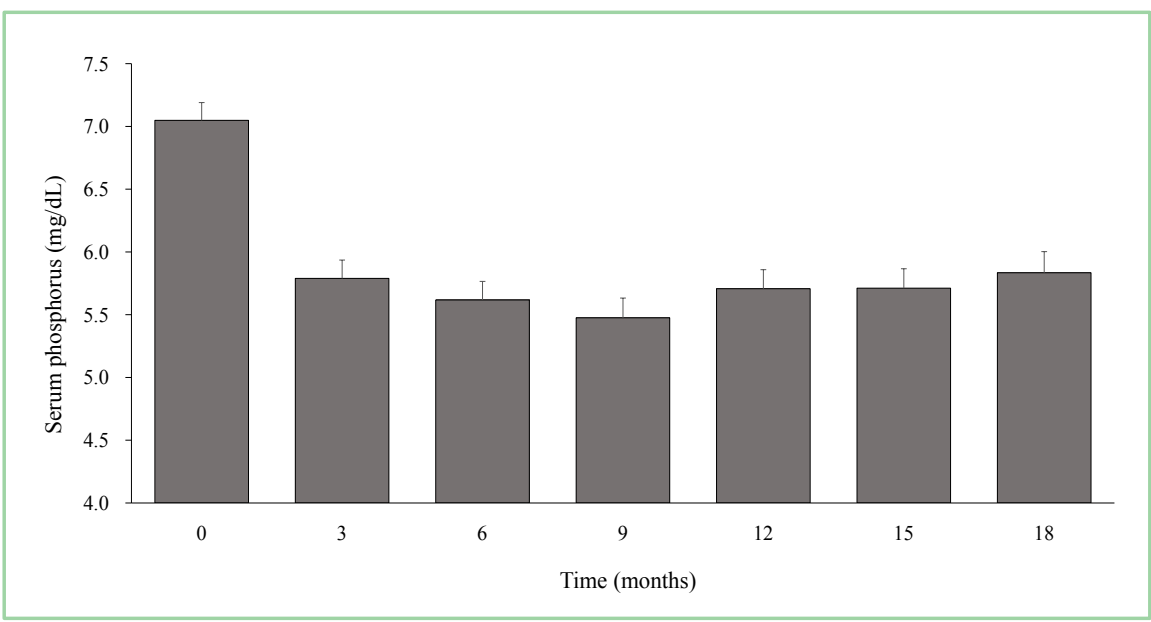

(b) 


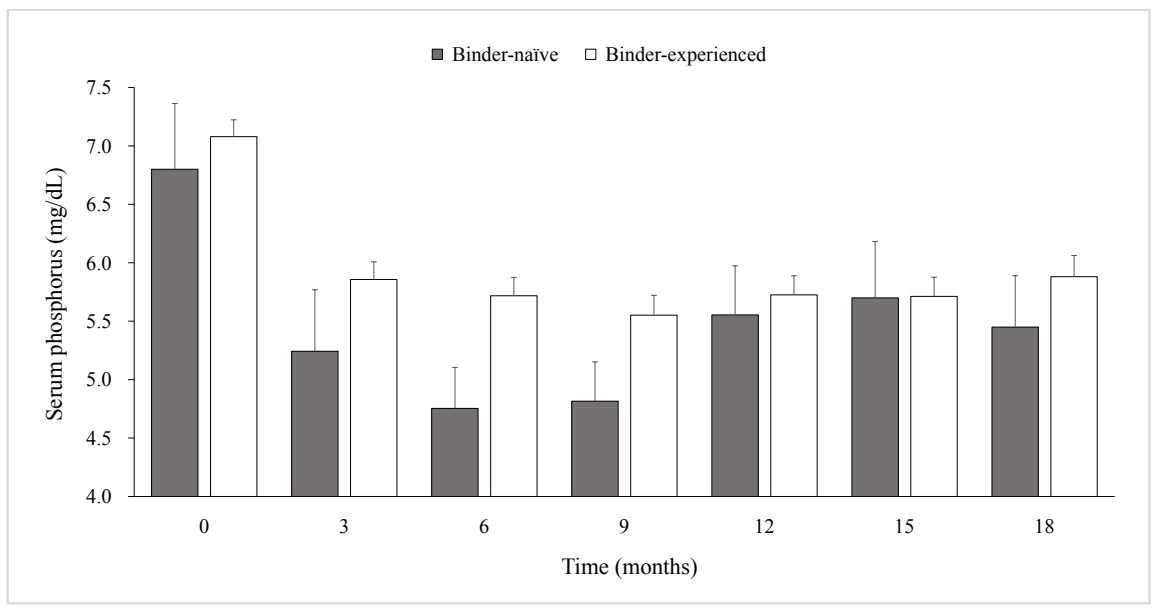

(c)

Figure 1. Serum phosphorus levels overall and by subgroup. Data presented are mean + SEM. (a) Percent of patients within KDOQI target serum phosphorus range of $\leq 5.5$ $\mathrm{mg} / \mathrm{dL}$. (b) Serum phosphorus levels over time for all patients. (c) Serum phosphorus levels for binder-experienced subgroup.

Table 3. Mean values of biomarkers at the start and end of the study.

\begin{tabular}{ccc}
\hline & \multicolumn{2}{c}{ Time (months) } \\
\hline & 0 & 18 \\
\hline $\mathrm{P} \leq 5.5 \mathrm{mg} / \mathrm{dL}$ & $18.8 \%$ & $47.7 \%$ \\
$\mathrm{P}>5.5 \mathrm{mg} / \mathrm{dL}$ & $81.3 \%$ & $52.3 \%$ \\
$\mathrm{P}$ mean \pm SEM & $7.05 \pm 0.14 \mathrm{mg} / \mathrm{dL}$ & $5.83 \pm 0.17 \mathrm{mg} / \mathrm{dL}$ \\
Binder-naïve & $6.80 \pm 0.56 \mathrm{mg} / \mathrm{dL}$ & $5.45 \pm 0.44 \mathrm{mg} / \mathrm{dL}$ \\
Binder-experienced & $7.08 \pm 0.14 \mathrm{mg} / \mathrm{dL}$ & $5.88 \pm 0.18 \mathrm{mg} / \mathrm{dL}$ \\
Hemoglobin & $11.96 \pm 0.76 \mathrm{~g} / \mathrm{dL}$ & $11.45 \pm 0.13 \mathrm{~g} / \mathrm{dL}$ \\
TSAT & $25.19 \pm 1.21 \%$ & $26.18 \pm 0.99 \%$ \\
Ferritin & $71.13 \pm 6.09 \mathrm{ng} / \mathrm{mL}$ & $124.94 \pm 8.62 \mathrm{ng} / \mathrm{mL}$ \\
ERI & $54.55 \pm 3.88 \mathrm{IU} / \mathrm{kg} /$ week & $52.90 \pm 5.79 \mathrm{IU} / \mathrm{kg} /$ week \\
ESA & $8411.36 \pm 561.05 \mathrm{IU} /$ week & $7873.66 \pm 740.88 \mathrm{IU} /$ week \\
\hline
\end{tabular}

Table 4. Changes in mineral bone biomarkers during the study period.

\begin{tabular}{ccccccccccc}
\hline \multicolumn{1}{c}{ Serum phosphorus $(\mathrm{mg} / \mathrm{dL})$} & \multicolumn{3}{c}{ Serum calcium $(\mathrm{mg} / \mathrm{dL})$} & \multicolumn{2}{c}{ iPTH } & \multicolumn{2}{c}{$(\mathrm{pg} / \mathrm{mL})$} \\
\hline Month & $\mathrm{N}$ & Mean & SEM & $\mathrm{N}$ & Mean & SEM & $\mathrm{N}$ & Mean & SEM \\
\hline 0 (Baseline) & 128 & 7.05 & 0.14 & 128 & 8.76 & 0.07 & 132 & 238.0 & 22.4 \\
3 & 127 & 5.79 & 0.15 & 127 & 8.79 & 0.07 & 130 & 208.8 & 21.7 \\
6 & 125 & 5.61 & 0.15 & 125 & 8.82 & 0.07 & 127 & 201.1 & 21.3 \\
9 & 126 & 5.48 & 0.16 & 126 & 8.76 & 0.06 & 126 & 201.6 & 22.3 \\
12 & 119 & 5.71 & 0.15 & 119 & 8.77 & 0.05 & 125 & 200.7 & 25.4 \\
15 & 116 & 5.71 & 0.16 & 116 & 8.76 & 0.06 & 121 & 200.1 & 23.4 \\
18 & 111 & 5.83 & 0.17 & 111 & 8.68 & 0.06 & 120 & 196.9 & 25.8 \\
\hline
\end{tabular}


The serum $\mathrm{Hb}(\mathrm{g} / \mathrm{dL})$, TSAT $(\%)$ and ferritin $(\mathrm{ng} / \mathrm{mL})$ levels were measured as the iron biomarkers. Changes in the levels of these biomarkers after 3 to 18 months of ferric citrate hydrate treatment are shown in Figure 2. The level of each of the iron biomarkers tended to increase during the first 3 months of treatment, with the levels remaining sustained at the elevated levels until the end of the study period, that is, 18 months of treatment.

The level of each of the iron biomarkers and the utilization of iron showed a significant change immediately after the start of the ferric citrate hydrate treatment. The serum Hb, TSAT, and ferritin levels were $11.96 \pm 0.76 \mathrm{~g} / \mathrm{dL}, 25.19 \pm$ $1.21 \%$, and $71.13 \pm 6.09 \mathrm{ng} / \mathrm{mL}$, respectively, at the baseline, and tended to decrease slightly after the start of the ferric citrate hydrate treatment; thereafter, they were sustained at the same levels until 18 months of treatment $(11.45 \pm 0.13$ $\mathrm{g} / \mathrm{dL}$ ) (Figure 2(a)). Similarly, the mean TSAT and serum ferritin levels were maintained at $26.18 \% \pm 0.99 \%$ (Figure $2(\mathrm{~b})$ ) and and $124.94 \pm 8.62 \mathrm{ng} / \mathrm{mL}$ (Figure 2(c)), respectively, until 18 months (Table 3).

Data on the monthly dose of erythropoietin stimulating agent (ESA) were collected at the same time points as the test data. The dose of ESA decreased after 3 months of ferric citrate hydrate treatment, but tended to increase slightly thereafter, and did not return to the baseline level until the end of the study period (Figure 3). The ESA resistance index (ERI) was determined as the weekly weight-adjusted dose of epoetin (IU/kg/week) devided by $\mathrm{Hb}$ concentration (g/dL), as previously described [7]. The changes in ERI are shown in Figure 4.

Seven patients dropped out of the study due to adverse drug reaction, such as diarrhea, but there were no AEs and serious AEs.

\section{Discussion}

In this prospective study of dialysis patients treated with ferric citrate hydrate, treatment with ferric citrate hydrate resulted in a sustained reduction of the serum $P$ level until the end of the study period of 18 months, and also a gradual reduction of the serum iPTH level, with no change of the serum Ca level. The number of patients in whom the serum $\mathrm{P}$ level reached the target range doubled ( $23 \%$ vs. $48 \%$ ) by 3 months after the start of the ferric citrate hydrate treatment, and became two-thirds of the total by 18 months of treatment. The effects of ferric citrate hydrate on the serum $\mathrm{P}$ level were comparable in the prior P-binder therapy(-) and prior P-binder therapy(+) subgroups.

Ferric citrate hydrate has been shown to increase the iron stores in CKD patients [8]. In this study, we analyzed the impact of ferric citrate hydrate treatment on the serum levels of iron biomarkers (Hb, TSAT and serum ferritin). The measured levels of these markers were maintained for 18 months, with an increase in the iron stores over time. As compared to the prior P-binder thera$\mathrm{py}(+)$ patients, the serum iron biomarker levels before the start of ferric citrate hydrate treatment were lower in the prior P-binder therapy(-) patients. However, after 18 months of treatment with ferric citrate hydrate, the iron biomarker levels were similar between the two subgroups. 
The data from this study were similar to those reported from the Phase III study of ferric citrate hydrate $\left(\right.$ Riona $\left.^{\oplus}\right)$. Fifty-two weeks after the start of the

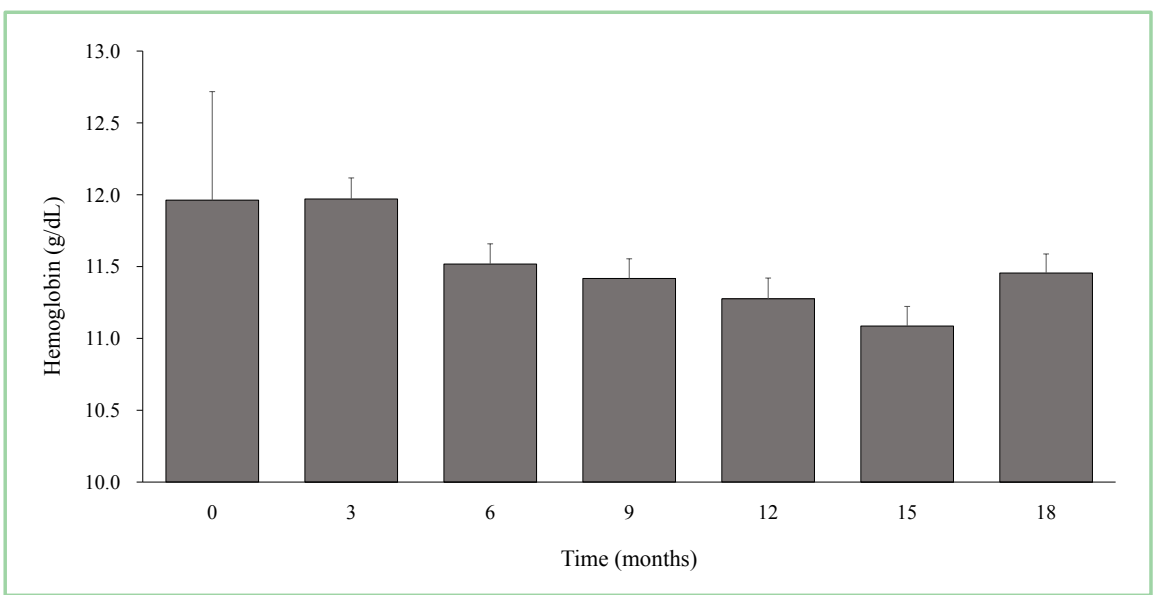

(a)

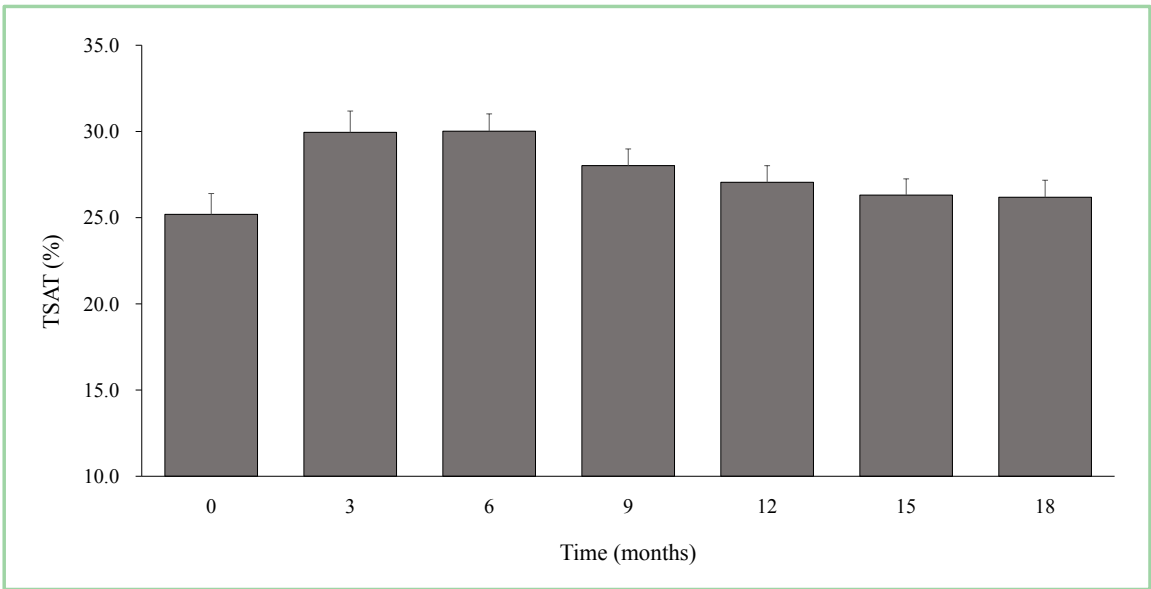

(b)

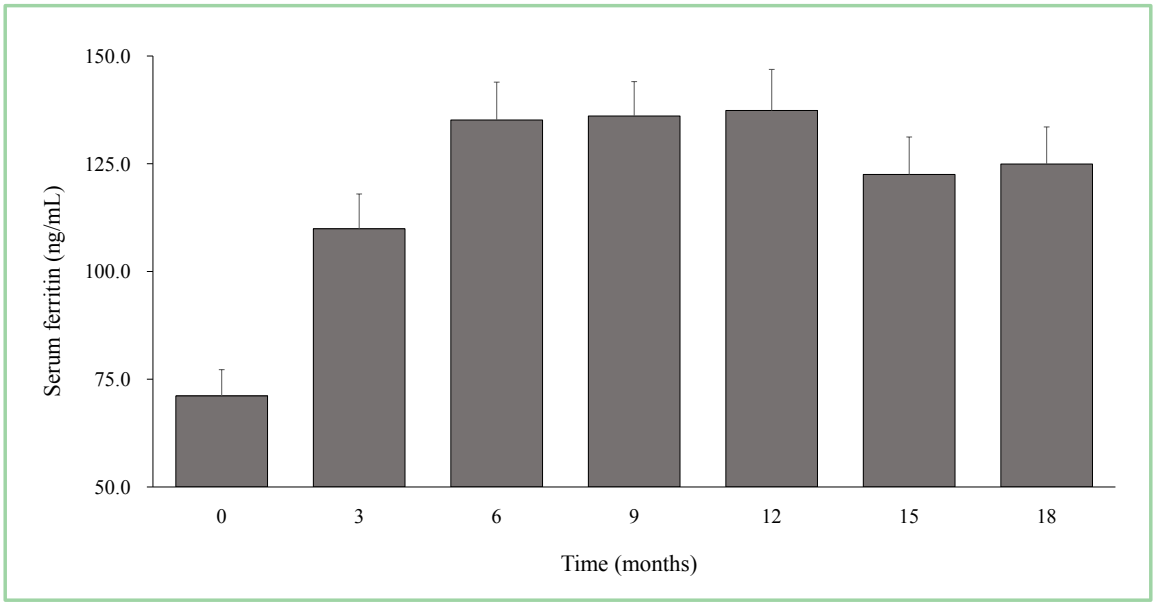

(c)

Figure 2. Mean (standard error of the mean) iron biomarker measurements over time. Data presented are mean + standard error of the mean. (a) Mean hemoglobin for all patients. (b) Mean TSAT for all patients. (c) Mean serum ferritin for all patients. 


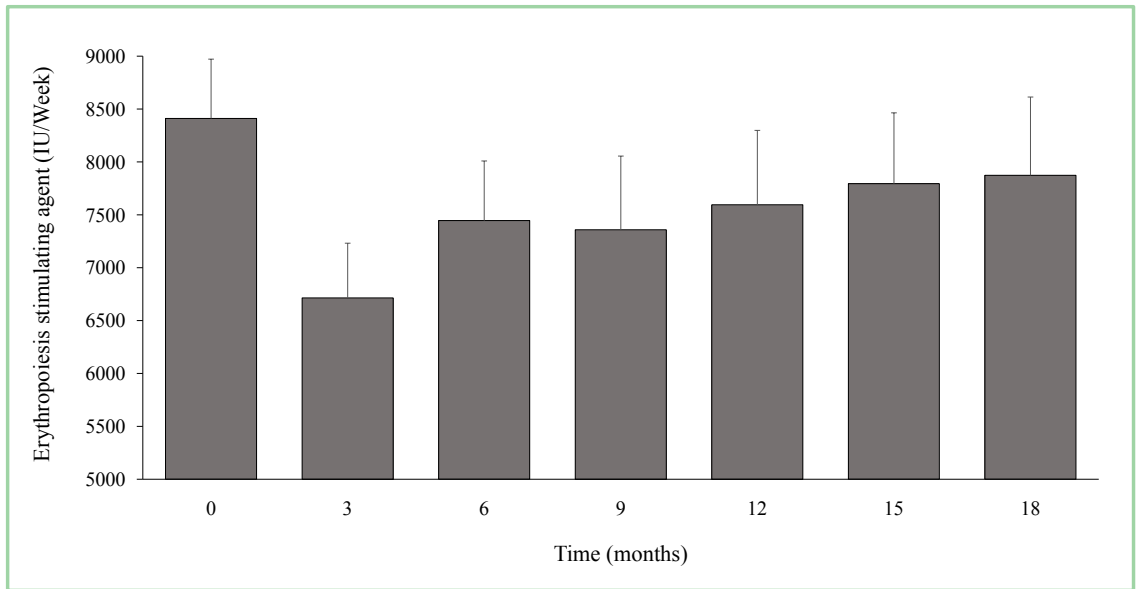

Figure 3. Changes in doses of erythropoiesis-stimulating agents over time.

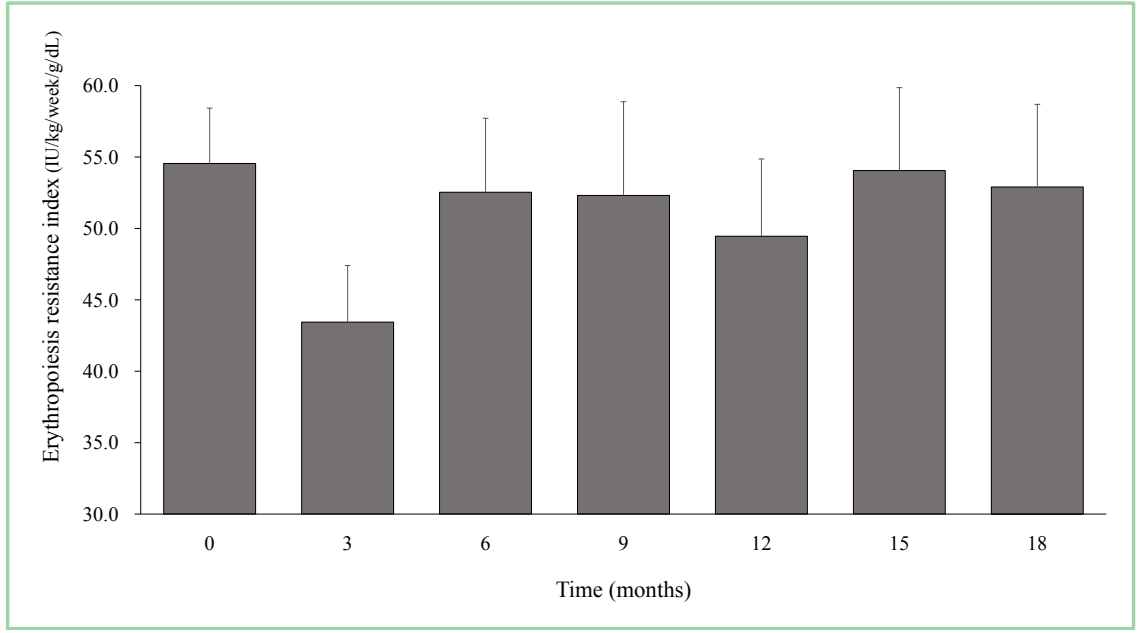

Figure 4. Changes in erythropoiesis resistance index over time.

Phase III active-controlled study, the mean serum $\mathrm{P}$ level in the patients randomized to ferric citrate hydrate became $5.36 \mathrm{mg} / \mathrm{dL}$, similar to the serum P level $(5.42 \mathrm{mg} / \mathrm{dL})$ seen in this study after 6 months of treatment. In addition, a similar trend was observed in the serum iron biomarker levels $(\mathrm{Hb}$, ferritin and TSAT) between the Phase III study ( $11.7 \mathrm{~g} / \mathrm{dL}, 899 \mathrm{ng} / \mathrm{mL}$ and 39\%, respectively) and this study $(11.1 \mathrm{~g} / \mathrm{dL}, 947 \mathrm{ng} / \mathrm{mL}$ and 37\%, respectively, after 6 months of treatment).

Moreover, the results of this study were also consistent with the results from the national sampling based on the "Dialysis Outcomes and Practice Patterns Study (DOPPS)" database [9]. The DOPPS Practice Monitor reports clinical follow-up data over time, using samples collected from more than 11,000 HD patients in more than $200 \mathrm{HD}$ facilities. Sixty-two percent to $72 \%$ of patients followed up by the DOPPS Practice Monitor from 2010 to 2015 showed serum P levels within the target range $(\leq 5.5 \mathrm{mg} / \mathrm{dL})$. After 6 months of treatment in this study, $65 \%$ of patients showed serum $\mathrm{P}$ levels within the target range. The slightly lower value of $65 \%$ in this study may be related to the patient selection 
criteria for this study: Many patients enrolled in this study were switched from their previous P-binder drug(s), because these were less effective or caused adverse drug reactions (only $22 \%$ of the patients had a serum P level within the target value at the baseline); some patients still showed poor compliance with the newly started P-binder treatment after the treatment switch; there may have been a persistent increase in the $\mathrm{P}$ levels as a result of unhealthy eating habits.

In regard to the iron markers, the DOPPS Practice Monitor reported that during the period from 2010 to 2015 , the TSAT was $\leq 29 \%$ in $50 \%$ to $60 \%$ of patients across the US, the serum ferritin level was $\leq 799 \mathrm{ng} / \mathrm{mL}$ in $55 \%$ to $70 \%$ of patients, and the serum $\mathrm{Hb}$ was $\geq 11.0 \mathrm{~g} / \mathrm{dL}$ in $41 \%$ to $72 \%$ of patients. Interestingly, these levels were similar to the mean values observed in our present study. The data were difficult to interpret because of the large variations in the dose of intravenous iron. However, the Phase III study in patients with end-stage renal failure demonstrated a reduction in both the intravenous iron dose and ESA use over the 52-week trial period.

Although this study had limitations, as mentioned below, we believe that use of real-world clinical data can provide valuable clinical information to physicians prescribing drugs. The limitations of this study were: 1) The sample size of the study was 132 , which is relatively small. However, this is the largest clinical study to date conducted on the use of ferric citrate hydrate in clinical practice; 2) the patients analyzed were limited to those who took ferric citrate hydrate for at least 3 months (data collected for 18 months). As a result, patients who could not tolerate ferric citrate hydrate and those who discontinued ferric citrate hydrate treatment for other reasons were excluded from the analysis. Despite this limitation, there were few selection criteria; therefore, we would like to add that there was almost no obvious selection bias in this study; 3) both patients who were already on hemodialysis and patients who were close to initiation of hemodialysis were included in the study; notwithstanding, the sample used in this study may represent the diversity of patients who receive ferric citrate hydrate in actual clinical settings; 4 ) as expected from the retrospective design of this study, the data collected were limited to the data obtained from the patients' medical records, and no specific compliance rates were included in the analysis. However, a post hoc analysis of the extremely important Phase III study comparing ferrate citrate hydrate with an active drug showed a mean treatment compliance rate of $81 \%$ for both the study drug and the active drug. Therefore, it is reasonable to assume that the compliance rate in this study was similar to that in the phase III study; 5) The limited data on the use and doses of the prescribed ESA and intravenous iron are due to the discrepancies in the ESA administration protocol among the medical institutions that participated in this study for 18 months. It may be worth investigating the association between ESA and intravenous iron use in patients receiving ferric citrate hydrate, as previously described [10]; 6) finally, it should be noted that no new safety concerns were identified in this study and there were no serious AEs. 


\section{Conclusion}

The clinical practice data used in this study showed that the target serum P level was reached in $65 \%$ of patients by 6 months after the start of ferric citrate hydrate treatment, demonstrating that ferric citrate hydrate is a P-binder that also contributes to increasing the iron stores, in general, and maintenance of the serum $\mathrm{Hb}$ level. In this study, no new safety concerns were identified.

\section{Acknowledgements}

The authors are very grateful to dialysis staffs of Jyoban Hospital.

\section{Conflicts of Interest}

The authors declare no conflicts of interest regarding the publication of this paper.

\section{References}

[1] Coladonato, J.A. (2005) Control of Hyperphosphatemia among Patients with ESRD. Journal of American Society of Nephrology, 16, S1107-S1114. https://doi.org/10.1681/ASN.2005060663

[2] Noordzij, M., Korevaar, J.C., Boeschoten, E.W., Dekker, F.W., Bos, W.J. and Krediet, R.T. (2006) Hyperphosphataemia and Related Mortality. Nephrology Dialysis Transplantation, 21, 2676-2677. https://doi.org/10.1093/ndt/gfl229

[3] Isakova, M., Gutierrez, O.M., Chang, Y., Shah, A., Tamez, H., Smith, K., Thadhari, R. and Wolf. M. (2009) Phosphorus Binders and Survival on Hemodialysis. Journal of American Society of Nephrology, 20, 388-396. https://doi.org/10.1681/ASN.2008060609

[4] Yokoyama, K., Hirakata, H., Akiba, T., Sawada, K. and Kumagai, Y. (2012) Effect of Oral JTT-751 (Ferric Citrate) on Hyperphosphatemia in Hemodialysis Patients: Results of a Randomized, Double-Blind, Placebo-Controlled Trial. American Journal of Nephrology, 36, 478-487. https://doi.org/10.1159/000344008

[5] Yokoyama, K., Akiba, T., Fukagawa, M., Nakayama, M., Sawada, K., Kumagai, Y., Chertow, G.M. and Hirakata, H. (2014) A Randomized Trial of JTT-751 versus Sevelamer Hydrochloride in Patients on Hemodialysis. Nephrology Dialysis Transplantation, 29, 1053-1060. https://doi.org/10.1093/ndt/gft483

[6] Uhlig, K., Berns, J.S., Kestenbaum, B., Kumar, R., Leonard, M.B., Martin, K.J., Sprague, S.M. and Goldfarb, S. (2010) KDOQI US Commentary on the 2009 KDIGO Clinical Practice Guideline for the Diagnosis, Evaluation, and Treatment of CKD-Mineral and Bone Disorder (CKD-MBD). American Journal of Kidney Diseases, 7, 3-6. https://doi.org/10.1053/j.ajkd.2010.02.340

[7] Okazaki, M., Komatsu, M., Kawaguchi, H. and Nitta, K. (2014) Erythropoietin Resistance Index and the All-Cause Mortality of Chronic Hemodialysis Patients. Blood Purification, 37, 106-112. https://doi.org/10.1159/000358215

[8] Yokoyama, K., Fukagawa, M., Akiba, T., Nakayama, M., Otoguro, T., Yamada, K., Nagamine, Y., Fishbane, S. and Hirakata, H. (2017) Ferritin Elevation and Improved Responsiveness to Erythropoiesis-Stimulating Agents in Patients on Ferric Citrate Hydrate. Kidney International Reports, 2, 359-365. https://doi.org/10.1016/j.ekir.2016.12.005

[9] Blayney, M.J. and Tentori, F. (2009) Trends and Consequences of Mineral Bone 
Disorder in Haemodialysis Patients: Lessons from the Dialysis Outcomes and Practice Patterns Study (DOPPS). Journal of Renal Care, 35, 7-13.

https://doi.org/10.1111/j.1755-6686.2009.00048.x

[10] Yokoyama, K., Fukagawa, M., Akiba, T., Nakayama, M., Ito, K., Hanaki, K., Wolf, M. and Hirakata, H. (2019) Randomised Clinical Trial of Ferric Citrate Hydrate on Anaemia Management in Haemodialysis Patients with Hyperphosphataemia: ASTRIO Study. Scientific Reports, 9, 8877. https://doi.org/10.1038/s41598-019-45335-4 